\title{
Fat Embolism Syndrome in a Patient with Bilateral Tibial Fractures: Report of the Case and Review of the Literature
}

\author{
Ilias Alexandros Kosmidis ${ }^{1^{*}}$, Konstantinos Kourkoutas ${ }^{1}$, Ioannis Bampalis ${ }^{1}$, \\ Panagiotis Giannakopoulos ${ }^{2}$ \\ ${ }^{1}$ Second Orthopaedic Department, KAT General and Trauma Hospital, Athens, Greece \\ ${ }^{2}$ Upper Extremity Surgery, Pittsburg University, USA and Athens Medical Center, Maroussi, Greece \\ Email: a.i.kosm@gmail.com, kourkoutasdoc@windowslive.com, ioannisbampalis@gmail.com, \\ pgiannak@otenet.gr
}

Received 12 August 2014; revised 29 September 2014; accepted 15 October 2014

Copyright (C) 2014 by authors and Scientific Research Publishing Inc.

This work is licensed under the Creative Commons Attribution International License (CC BY). http://creativecommons.org/licenses/by/4.0/

(c) (i) Open Access

\begin{abstract}
Background: Although the original clinical description of fat embolism syndrome (FES) dates from 1873, the condition remains a diagnostic challenge for modern clinicians. The syndrome is described as a serious consequence of fat emboli producing a distinct pattern of clinical symptoms and signs. It is mainly associated with fractures of the long-bones and the pelvis. The present paper describes the case of a trauma patient with bilateral tibial fractures that present the syndrome and highlights the importance of early identification and aggressive treatment of FES which is obligatory for the patient's survival. Case Description: A 32 year-old man reached the hospital as a trauma call after a road traffic accident. Clinical examination was unremarkable with the only finding that the patient was unable to straight leg raise and weight bear. Both shins were painful and swollen at their middle third, while the left one was in varus and posterior displacement. Neurovascular status of both lower extremities was normal. Plain radiographic control (X-Rays) revealed an oblique right tibial fracture of the mid-shaft with a distal third fibular fracture and a comminuted fracture of the left tibia with a fracture of the fibula at the same level. The legs were immobilised on a splint and the decision was to treat the patient surgically. Within a few post admission hours the haemoglobin dropped by $2.9 \mathrm{mg} / \mathrm{dL}$, however a source of active bleeding could not be allocated. The abdomen was soft and not tender in palpation and the central nervous system did not present any abnormalities. The following day the haemoglobin dropped but the new ultrasonography control did not reveal any free abdominal fluid. Both lower limbs were not compromised. The second post-injury day the patient went to theatre and the right tibia was stabilised with an antegrade nail (T2-Stryker) while the left one with an external fixation. During the reaming proc-
\end{abstract}

${ }^{*}$ Corresponding author.

How to cite this paper: Kosmidis, I.A., Kourkoutas, K., Bampalis, I. and Giannakopoulos, P. (2014) Fat Embolism Syndrome in a Patient with Bilateral Tibial Fractures: Report of the Case and Review of the Literature. Open Journal of Orthopedics, 4, 273-284. http://dx.doi.org/10.4236/ojo.2014.410044 
ess the haemoglobin dropped to $7.1 \mathrm{gr} / \mathrm{dL}$, so he was transfused with 3 blood units. Immediately after extubation, the patient desaturated on air and ABG values forced the anaesthetists to intubate him in recovery and place him in mechanical ventilation with high levels of positive end expiratory pressure (PEEP). Symptoms subsided rapidly and he was transferred to the IMU. Clinical image and CT scan were in favour of fat embolism syndrome. On the fifth post-operative day the patient was transferred back to ward and a week later he was discharged being completely free of symptoms. Clinical Relevance: Fat embolism syndrome is typically presented with the following triad: a) respiratory changes; b) neurologic abnormalities; c) petechial rash. These three constitute the major criteria described by Gurd and are pathognomonic for the condition. The patient-described in the present case report-developed progressively all those symptoms within the expected period of time. The FES is a well-known pathological condition that is well described in literature and should be familiar to those clinicians that manage trauma patients. Diagnosing the condition can be highly demanding since there are no laboratory investigations or radiographic imaging techniques specific for FES. Suspicion and diagnosis can be mainly placed based on the criteria described by Gurd, in 1970.

\section{Keywords}

Fat Embolism Syndrome, Long Bone Fractures, Respiratory Distress Syndrome and Embolism of Fat

\section{Background}

Fat embolisation can describe two different conditions with completely altered clinical impact. These can be either the circulation of fat in blood stream, or expression of the syndrome itself. Those two conditions are independent and not related to each other; fat in the circulation is not prerequisite for expression of fat embolism syndrome. It is helpful to define each entry, acknowledging that there may be some overlap in clinical practise. Fat embolism (FE) is fat in the circulation that can produce embolic phenomena, presenting or not from clinical sequelae. On the other hand, fat embolism syndrome (FES) is fat in the circulation associated with an identifiable clinical pattern of symptoms and signs.

The subject of FES is a popular and of recurring interest to those managing trauma. However, despite advances in imaging and laboratory investigations, the occurrence rate still remains high. The purpose of the article is to cover the syndrome in general, including diagnosing methods and treatment options, and to describe the case report of a trauma patient, with two long-bones fractures that developed FES, in order to point out the primary role and importance of clinical expertise in the successful management of the condition. And the adjuvant investigations have a supportive secondary role.

\section{Case Report}

A 32 year-old male motorcyclist was involved in a road traffic accident (RTA) and presented to our hospital as a trauma call. The patient maintained consciousness; vital signs were within normal range (blood pressure was $120 \mathrm{mmHg}$ over $85 \mathrm{mmHg}$, heart rate 75/min, apyrexial and respiratory rate $18 / \mathrm{min}$ ) while clinical examination of the spine, chest and abdomen was unremarkable. The abdominal sonography for trauma-initially performed - did not reveal free intra-abdominal fluid. Both shins were painful and the patient was unable to weight bear. He could not straight leg raise due to significant pain at the mid-shaft of both tibias. Examination of the right shin revealed swelling of the mid shaft, soft tissue bruising and lacerations of the skin. The left shin was swollen too and the distal third was in varus and posteriorly deformed. Both limbs were not compromised (distal Pulses were palpable) at the time and neurological examination was normal. Routine blood tests including full blood count, biochemistry, clotting, group and cross matching were performed and values were found within normal rates.

Routine radiographic examination of the patient included X-Rays of the C-spine, chest, pelvis and the tibias. Remarkable findings were only from the lower limbs where the anteroposterior (Figure 1(a)) and lateral views 
(Figure 1(b)) showed an oblique right tibial fracture of the mid shaft combined with a fracture of the fibula at the distal third and a comminuted oblique fracture of both the left tibia and fibula of the mid third. Both legs were placed in an above knee back slab, anti-coagulant treatment with low- molecular-weight heparin (Dalteparin) started and the patient was transferred to the ward in order to be scheduled for surgical management of his fractures.

Within the next eight post-admission hours, haemoglobin dropped by $2.9 \mathrm{mg} / \mathrm{dL}$ (initial Hb was $13.1 \mathrm{mg} / \mathrm{dL}$, four hours later dropped to $11.6 \mathrm{mg} / \mathrm{dL}$ and after eight hours to $10.2 \mathrm{gr} / \mathrm{dL}$ ) and haematocrit from $40.4 \%$ to $35.4 \%$. Clinical examination could not justify a source of haemorrhage; the abdomen was soft, not tender in palpation, there were no findings from the chest and the central nervous system (CNS) did not present any abnormality. An urgent abdominal CT scan was requested in order to identify the haemorrhagic source but the results were negative for bleeding. During the night the patient developed a pyretic wave of $38.0^{\circ} \mathrm{C}$ that was not evaluated further.

The following day the patient was clinically stable, however haemoglobin dropped to $10.1 \mathrm{gr} / \mathrm{dL}$ and the haematocrit (Hct) to $30 \%$. A new abdominal ultrasonography was performed that was negative for free abdominal fluid. The decision was not to transfuse the patient. Both splints were opened and neurovascular status of the limbs was thoroughly checked. Pulses were palpated all the way down to the forefoot; both shins were found swollen while additional blisters were formed above the fractured area of the left leg. The limbs were not compromised and the plan was to treat the fractures by using an antegrade tibial nail (T2-Stryker) for the right tibia and an external fixation for the left, more comminuted, one. The patient was found fit for surgery by the anaesthetists, so the second post-injury day he went to theatre for fixation of his fractures.

Operation was performed under general anaesthesia. Nailing preceded external fixation. Limb tourniquet was not used during the nailing procedure. Reaming of the right tibia, nail insertion, distal and proximal locking of the nail were performed uneventfully. The external fixation was placed to the left tibia; three pins were used proximally and three other distally, all apart from the fractured area and the bruised, blistered and oedematous skin (Figure 2(a) and Figure 2(b)). During the operation, and mainly after the reaming process, the haemoglobin dropped further to $7.1 \mathrm{gr} / \mathrm{dL}$ (Hct 21\%); at that point the anaesthetists decided to transfuse the patient with 3 units of blood. At the end of the operation the haemoglobin and haematocrit value restored to $11.9 \mathrm{mg} / \mathrm{dL}$ and $35 \%$ respectively.

Immediately after extubation the patient desaturated rapidly on air with the following arterial blood gas

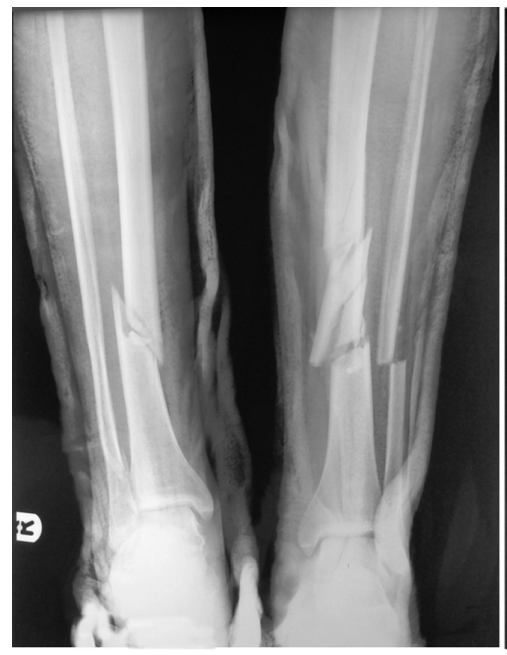

(a)

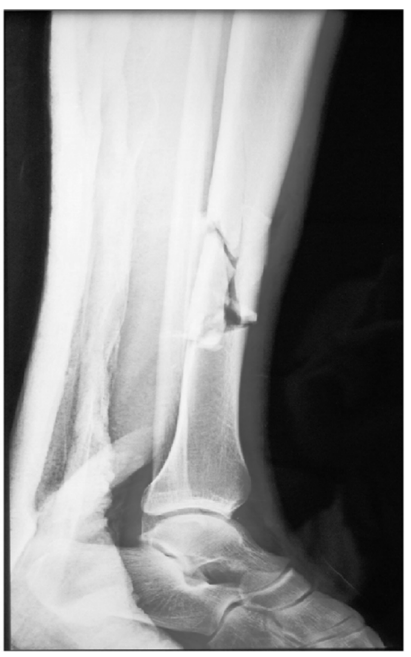

(b)

Figure 1. Plain pre-operative X-Rays of both tibias, stabilised on an above the knee splint: (a) the left X-Ray is an anteroposterior view showing a short oblique fracture of the distal third of the right tibia, with a Weber C fracture of the ipsilateral fibula and a comminuted fracture at the same level of the contralateral (left) tibia combined with a transverse fracture of the fibula at the same level; (b) The lateral X-Ray shows the comminuted right tibia, which is minimally displaced. 


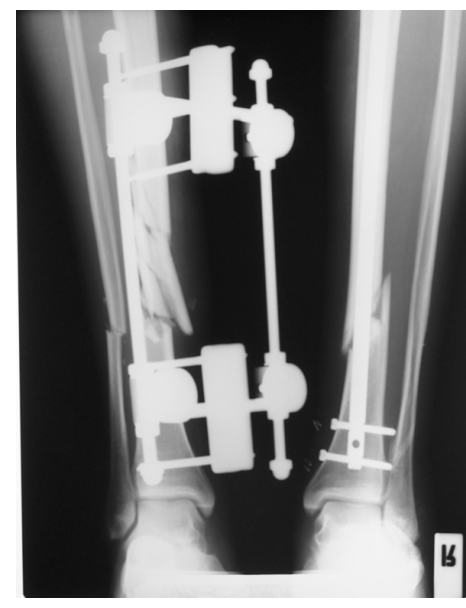

(a)

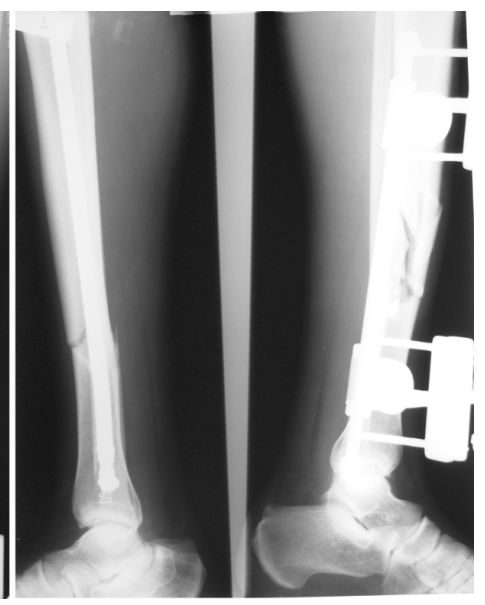

(b)

Figure 2. Post-operative radiographic control: (a) the X-Ray on the left, is an anteroposterior view that shows the IM tibial nail placed in the right tibia, which is locked distally with two screws, and the external fixation which is placed on the left comminuted tibia. Three pins are placed proximally to the fracture and three more distally; (b) on the lateral views both fractures are efficient reduced.

(ABG) values: $\mathrm{pO}_{2} 28.7 \mathrm{mmHg}, \mathrm{pCO}_{2} 48.3 \mathrm{mmHg}$ and $\mathrm{pH}$ 7.271. Findings from the chest were compatible with pulmonary oedema. Following ABG values, on a venturi mask, were $\mathrm{pO}_{2} 110 \mathrm{mmHg}, \mathrm{pCO}_{2} 58 \mathrm{mmHg}$ and $\mathrm{pH}$ 7.221. The patient was tachycardic, respiratory rate was $35 / \mathrm{min}$, no focal neurology was found and he was responsive to verbal stimuli. The anaesthetists decided to intubate the patient in recovery. He was placed in mechanical ventilation with high levels of positive end expiratory pressure (PEEP). Examination of the chest revealed immediate absorption of the lung fluid and improvement of the $\mathrm{ABG}\left(\mathrm{pO}_{2} 124 \mathrm{mmHg}, \mathrm{pCO}_{2} 51, \mathrm{pH}\right.$ 7.267). The ECG performed at that time showed SR tachycardia with $S_{1}$ and $Q_{3} \cdot S_{3}$ findings that rose suspicious of pulmonary embolism. Urgent radiographic control of the chest and head was performed including plain anteroposterior chest radiograph and CT scan of the head and the chest. Upon stabilisation of the patient's vital signs and respiratory function, he was transferred to the intensive care medical unit (IMU).

Radiographic findings were interesting. Plain chest X-Ray (Figure 3) showed bilateral fluffy shadows and diffuse airspace consolidation due to oedema oralveolar haemorrhage. Focal areas of ground glass opacification with interlobular septal thickening and multiple bilateral nodular lesions were seen in the chest CT scan (Figure 4(a) and Figure 4(b)). Imaging of the brain was normal. The most favourable diagnosis, based on clinical and laboratory findings (hypoxaemia, respiratory failure and drop of haematocrit and haemoglobin) strongly supported by radiographic and imaging findings, was fat embolism syndrome (FES).

On the second post-operative day, haemoglobin dropped further down. An active source of bleeding could still not be allocated. At that time subconjunctival and axillary petechiae (Figures 5(a) and Figure 5(b)) appeared which constitute one of the major diagnostic criteria for the FES. The patient was still under mechanical ventilation (PEEP) and ABGs were stabilised. The new chest X-Ray is compatible with the patient's clinical improvement; bilateral shadows disappeared and congestion subsided.

The day after (3rd post-op day) the young man had significantly improved. The haemoglobin and the haematocrit were stabilised and ABGs were normalised despite the reduction of PEEP. Findings from plain chest $\mathrm{X}$-Ray (Figure 6) were consistent with the clinical improvement. At that time he was extubated and remained in the IMU for two more days only for observation. On the 5th post op day the patient returned back to the ward; thromboprophylaxis with Dalteparin continued and gradual, non weight bearing, mobilisation with the physiotherapists was encouraged.

A week later he was discharged from the hospital, being completely asymptomatic from both the respiratory system and the surgical point of view. Anticoagulant treatment with Dalteparin —one injection daily — was administered for 6 weeks in total. In monthly follow up chest is clear and he is full weight bearing on the right leg, while he is partially weight bearing on the right one. 


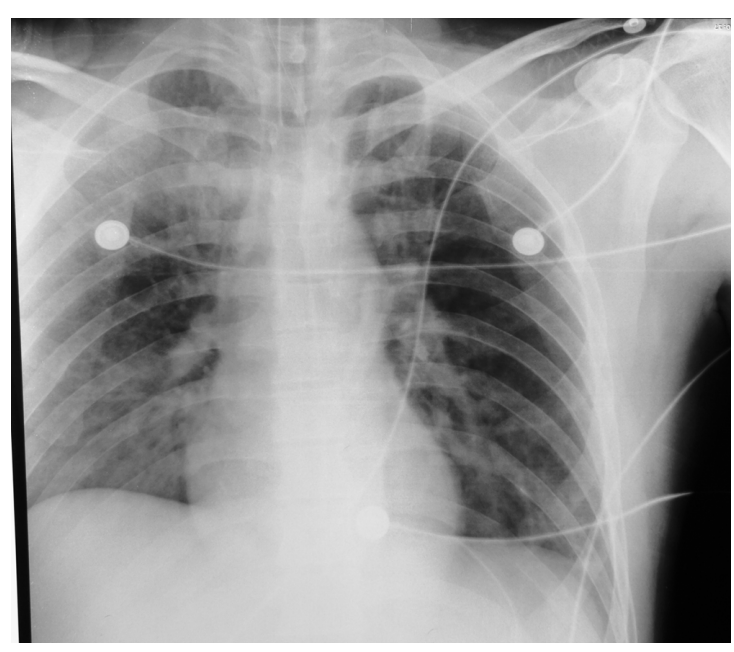

Figure 3. Plain anteroposterior X-Ray of the chest performed in supine position in the IMU. Radiographic findings are bilateral fluffy shadows and diffuse airspace consolidation.

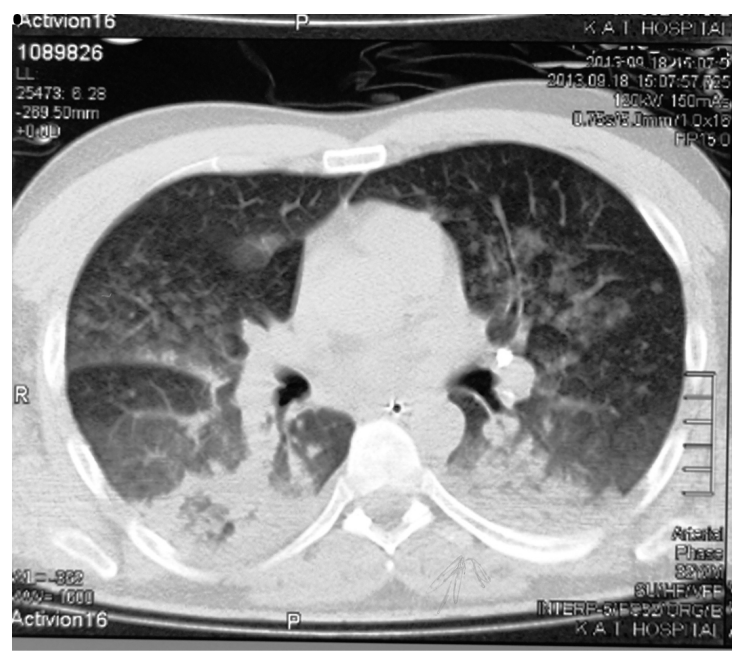

(a)

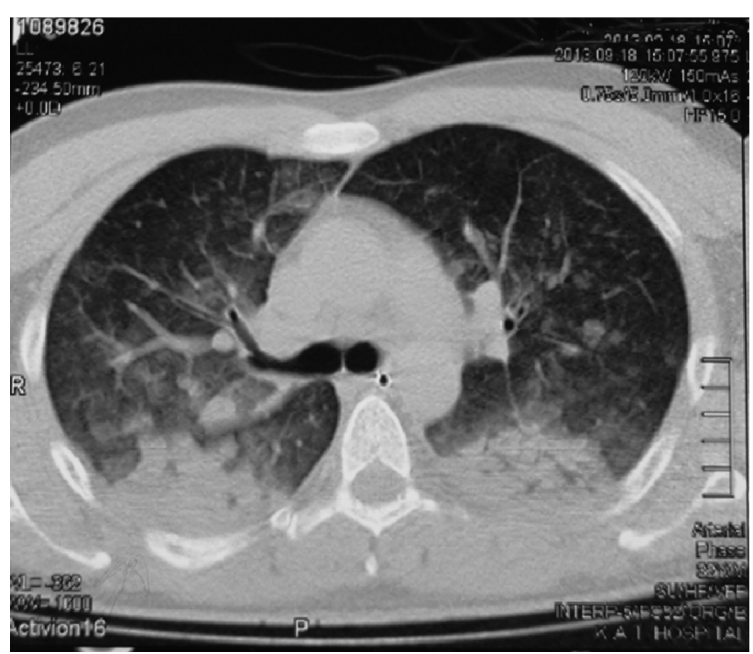

(b)

Figure 4. HR-CT chest scan in the IMU: (a) bilateral oedema at the bases of the lungs; (b) slices at lower level demonstrate ground-glass opacities confined to some lobules with a sharp margin between areas of involved and not involved lung. Also smooth, bilateral nodular lesions are present.

\section{Discussion}

Fat embolism is a well-known condition, described originally by Lower in animal model, around 330 years ago, who injected intravenous milk into dogs [1]. A few years later, Magendie observed that intravenous injection of oil, led to mechanical obstruction of small vessels by fat globules. Virchow further reported that intravenous injection of oil produced pulmonary oedema.

The original clinical description is dated back in the 1862 when Zenker described the first human case of post-traumatic embolism. Post mortem autopsy revealed the presence of fat globules in the pulmonary capillaries [2].

Fat embolism syndrome (FES) is a pathological condition which is not well defined by the international scientific community, therefore distinction between the phenomenon and the syndrome should be performed. Fat embolism (FE) is fat within the circulation, which can produce embolic phenomena followed or not by clinical sequel. On the contrary, FES is fat in the circulation associated with a specific clinical pattern of symptoms and signs from different systems, resulting from embolic showers. Despite the long history of that condition the syn- 


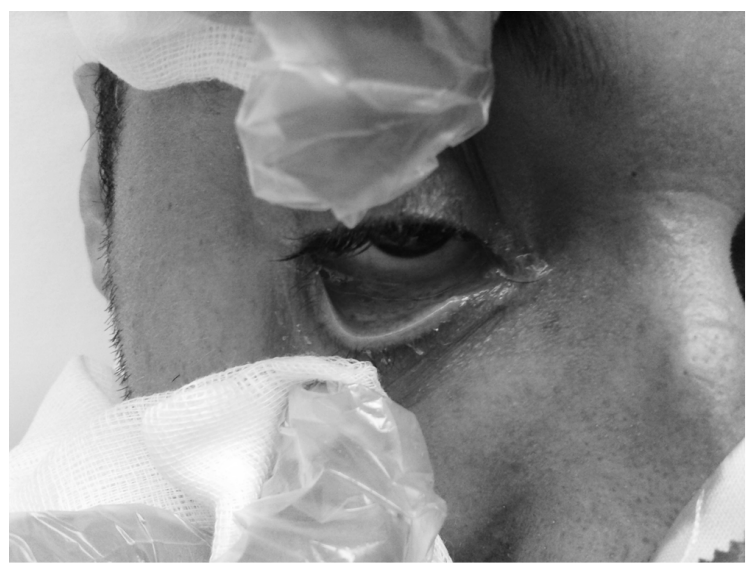

(a)

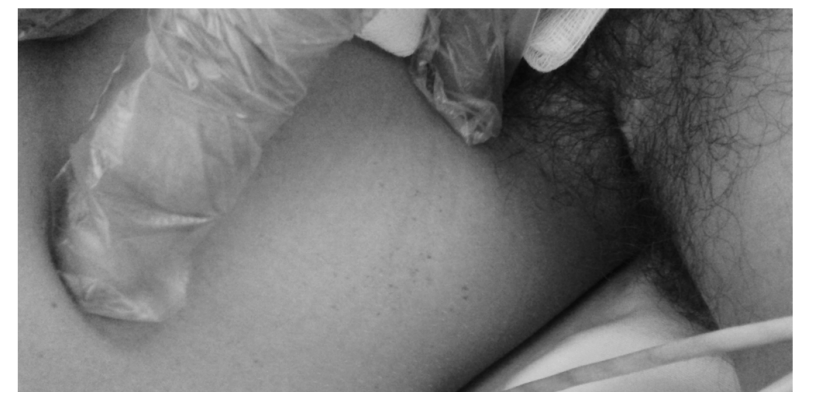

(b)

Figure 5. Petechial rash appeared on the second post-operative day. Subconjunctival (a) and axillary petechiae (b) are obviously seen on the photo.

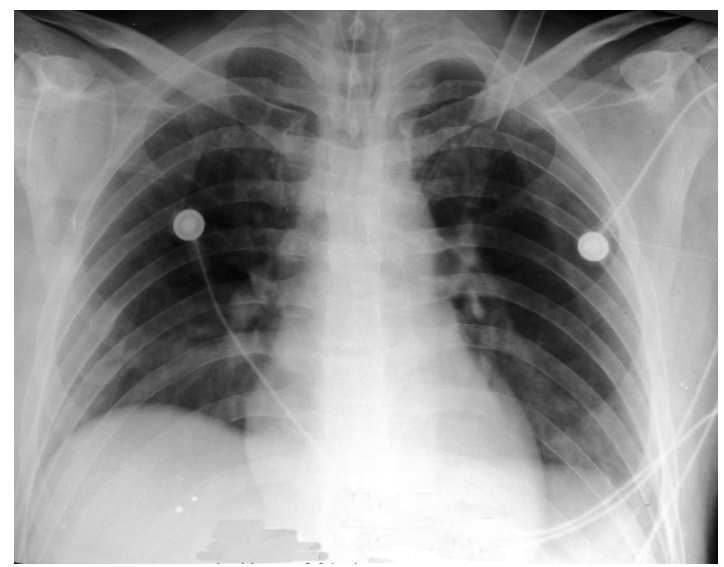

Figure 6. Anteroposterior radiograph of the patient's chest 72 hours after symptoms occurred. Both lungs are clear and there are no more signs of oedema; symptoms were completely reversed.

drome itself remains a diagnostic challenge for modern clinicians.

\subsection{Epidemiology}

Embolisation of fat is common among patients with orthopaedic trauma, especially in those with pelvic and long bone fractures [3]. The incidence of FES is accounted to $3 \%-4 \%$ in patients with isolated long bone trauma and $10 \%-15 \%$ in poly-trauma patients. As far as it regards the morbidity of the condition - directly attributable to FES-is dropped down to $10 \%$ thanks to modern intensive care units. Patients that survive from FES recover completely.

\subsection{Pathogenesis}

The mechanism that generates the syndrome is not well understood; explanation is based on two different theoretical models. Mechanical and biochemical causes have been proposed to be responsible for FES.

The mechanical theory suggests that fat, from disrupted bone marrow or adipose tissue, is forced into blood stream through the torn venues in the trauma area [4]. Furthermore, intramedullary surgical approaches are associated with higher intra canal pressures and with more fat emboli than those caused by external fixation techniques [5] [6]; normal marrow pressure is $30-50 \mathrm{mmHg}$ and during the reaming process can reach up to 600 
mmHg. The fact that most emboli occur during manipulations in the intramedullary cavity is verified ultrasonographically [7]. The importance of intramedullary fat in the FES is supported by the observation that previously reamed femurs are associated with significantly lower incidence of FES-related issues because of reduced intramedullary fat by the primary reaming process [8]. Cemented prostheses are also associated with higher rates of FE than the non-cemented ones, which however don't have minimal complications [9]. A disadvantage of that theory is the weakness to explain the $24-72 \mathrm{~h}$ delay in the development of the syndrome after the acute injury.

The second theoretical model is based on alterations in biochemical pathways that give arise to FES. One of those models involves the toxic effects of free fat acids (FFAs) on the vascular system; haemorrhagic oedema and destruction of the pulmonary anatomy follows the next 6 post injury hours [10]. Despite that neutral fatthe main bone marrow constitutes - is not that active, in vivo hydrolysis to FFAs may be a possible explanation to the interval between the injury and the symptoms arise. Beside the pulmonary effects of FFAs, studies have shown that they are involved in cardiac contractile dysfunction, another FES feature.

\section{Diagnosis}

\subsection{Clinical Presentation}

Fat embolism syndrome presents as a sequel of clinical symptoms and signs; however radiographic changes and biochemical values may be of value. The syndrome may present gradually with primary symptoms of hypoxaemia, central nervous system (CNS) suppression, hyperpyrexia and petechial rash. Time of presentation may vary between 12 - 36 post injury hours or can also occur intra-operatively [11]. The rash is considered pathognomonic of the FES and when presented diagnosis is established easier, however it is seen in only $20 \%-50 \%$ of cases [12].

In 1970, Gurd [13] proposed clinical criteria (Table 1) for the diagnosis of FES that still maintain their value and are widely used. These are divided into three major and eight minor criteria and the diagnosis of the syndrome is established when at least one major and four minor criteria are present.

The reliability and accuracy of Gurd's criteria has been questioned and other schemes based mainly on respiratory parameters have been proposed by different authors. Lindeque [14] raised a dispute and pointed out that Gurd's criteria may underdiagnose the syndrome. On the contrary, Lindeque's diagnosis of the syndrome was based mainly on respiratory features. More recently, Schonfeld [15] proposed a fat embolism index that gathers point from seven clinical features; each one gives a specific score (Table 2). The diagnosis of FES is placed with a score of 5 or more. However, the author acknowledged limitations of his system in patients suffering from cerebral, thoracic and abdominal trauma.

The classic triad of FES's presentation is pathognomonic and consists of

i. Respiratory changes;

ii. neurological abnormalities;

iii. petechial rash.

Pulmonary function impairment is among the dominant and primary features and is found in $75 \%$ of patients with fat embolism syndrome [16]. Early findings of the condition can be dyspnoea, tachypnoea and hypoxaemia

Table 1. Gurd's criteria for FES diagnosis.
\begin{tabular}{ll}
\hline Gurd's criteria for FES: 1 major +4 minor \\
\hline \multirow{3}{*}{ Major criteria } & Respiratory symptoms plus bilateral signs with positive radiographic changes \\
& Central nervous system depression disproportionate to hypoxaemia \\
& Petechial rash \\
\hline & Tachycardia $<110 \mathrm{bpm}$ \\
& Pyrexia \\
& Retinal fat or petechiae \\
& Urinary fat globules \\
Minor criteria & Sudden drop of Hg-level \\
& Sudden thrombocytopenia \\
& High erythrocyte sedimentation rate \\
& Fat globules in sputum
\end{tabular}


Table 2. Fat embolism index proposed by Schonfeld [15]. A diagnosis of FES is made with a score of 5 or more.

\begin{tabular}{cc}
\hline \multicolumn{1}{c}{ Points } & \\
\hline Diffuse petechiae & 5 \\
Alveolar infiltrates & 4 \\
Hypoxaemia & 3 \\
Confusion & 1 \\
Fever $>38^{\circ} \mathrm{C}$ & 1 \\
Heart rate $>120 / \mathrm{min}$ & 1 \\
Respiratory rate $>30 / \mathrm{min}$ & 1 \\
\hline
\end{tabular}

however the severity of lung dysfunction can vary from mild hypoxia - that can be controlled with supplemental oxygen - to respiratory failure that develops a syndrome similar to the acute respiratory distress syndrome (ARDS) that may require mechanical ventilation. Bulger et al. [17] studied 27 patients with established FES and reported that $44 \%$ required pulmonary support with mechanical ventilation. King et al. [10] reported that almost half of the patients with fat embolism syndrome, originated by long bone fractures, developed severe hypoxaemia and respiratory failure that required mechanical ventilation.

Neurological manifestations are also frequently seen in the early stages of the syndrome (FES). Cerebral embolisation by the fat globules produces neurological symptoms in up to $86 \%$ of the patients and usually follows the respiratory failure [18]. Another theory interprets the symptoms - not based on the ischaemia — but on the cerebral oedema [19]. Regardless the theoretic substance, a wide spectrum of symptoms is seen, from mild confusion and drowsiness to seizures, decortication or unresponsiveness [20]. However the most common presentation is with acute confusion. Focal neurology like hemiplegia, aphasia, apraxia, disturbances of the visual field and anisocoria have also been described. A case report of central nervous system involvement in the absence of pulmonary manifestations has been described. In that case, other diagnostic criteria were petechial rash, fever, tachycardia and hypotension [21]. In most cases neurologic deficiency is transient and fully reversible.

Based on the sequence of clinical appearance, petechial rash is the third major diagnostic sign. It is pathognomonic for the condition and is found in almost $60 \%$ of the patients [22]. Anatomical locations of appearance are the conjunctiva, the oral mucous membranes and skin folds of the upper body especially the neck and the axilla [23]. The distribution can be explained by the fat droplets that accumulate in the aortic arch prior to embolisation to non-dependent skin via the subclavian and carotid vessels [24]. Various factors contributing to the rash may be stasis, loss of clotting factors and platelets and endothelial damage from free fatty acids leading to rupture of the thin-walled capillaries [25]. The rash appears within the first 36 hours, is self-limiting and disappears completely within 7 days.

Apart from the diagnostic triad, mentioned above, toxic mediators - released either from initial injury or from lipid metabolism dysfunction - generate a number of minor features like as hyperpyrexia, tachycardia, myocardial depression, right heart strain ECG changes, coagulation abnormalities [26], renal dysfunction expressed as oliguria, lipiduria, proteinuria or haematuria. Moreover thrombocytopenia and an unexplained anaemia can be found in up to $37 \%-67 \%$ of the patients with FES [17].

A number of radiological findings have been described in FES but none of those is diagnostic for the condition. The plain chest X-ray is found normal in the first stages of the condition but later, when pulmonary function worsens, bilateral fluffy shadows, diffuse or patchy air space consolidation-due to oedema oralveolar haemorrhage - most prominent in the middle and upper zones have being observed and are described as "snow storm appearance". Chest CT depicts focal areas of ground glass opacity with interlobular septal thickening, centrilobular and subpleural nodules representing alveolar oedema, micro haemorrhage and inflammatory response secondary to ischaemia and cytotoxic emboli [27].

\subsection{Laboratory Investigations}

A wide range of investigations have been proposed for diagnosing FES, however none of those is absolutely specific. The most reliable and with highest diagnostic value test for FES is the measurement of arterial blood 
gases $(A B G)$. In any poly-trauma patient ABG measurement is essential as soon as possible and routine repetition should be performed within the next 48 post-injury hours.

Other laboratory investigations that may support the diagnosis are thrombocytopenia-observed in $37 \%$ of the patients (pathologic values of thrombocytes less than $150,000 \mathrm{ml}^{3}$ ) - and unexplained anaemia seen in $67 \%$. A number of additional laboratory findings have been related to FES but their appearance in other conditions apart from trauma cannot make them specific and reliable markers for FES. These include lipiduria, which is more frequently seen in up to $50 \%$ of the patients with significant bony injury, fat in the sputum, elevated serum lipase and phospholipase $\mathrm{A}_{2}\left(\mathrm{PLA}_{2}\right)$ measured in about $50 \%$ of patients with fractures and detection of droplets in the blood stream [28].

\subsection{Prevention and Treatment of FES}

The management of FES is based on three pylons: prevention, damage control and early diagnosis and adequate supportive and symptomatic measures.

In the attempt to prevent FES, modern surgeons face a significant difficulty; the absence of a universally accepted protocol. The chance of a patient to develop FES is a complex issue based on various factors as: the severity of the initial trauma, its anatomical location, the patient's genetic predisposition to an exaggerated inflammatory or coagulative response and finally the surgical management of his fractures. In most cases, insufficiency of the respiratory system can be predicted from the severity of the injury sustained [29]. Factors like an injury severity score (ISS) of over 16, a femoral fracture, a combined injury of the abdomen and the extremities and an unstable patient at the admission time, can independently predict with high assurance FES. On the same approach an ISS of less that 9 is very unlikely to lead to respiratory distress syndrome. Those observations have low positive predictive value and usually fail to identify those particular patients that develop respiratory problems after innocuous injury.

Prevention of the condition starts from the location of injury, the patients' transportation and arrival to the A+ E department (pre-hospital care). Of paramount importance is rapid and aggressive resuscitation and stabilisation in order to minimise the stress response and the hypovolaemia [30]. An effort to distinguish patients with higher possibility of developing FES is based on haematological markers or scores of vascular fat embolism. The most promising one seems to be interleukin-6 (IL-6); normal blood levels are less than $10 \mathrm{pg} / \mathrm{ml}$ and do not rise significantly after minor skeletal injuries, as an ankle fracture [31]. Pape et al. (2001) have shown that the levels of IL-6 rise significantly after trauma and are proportional to the extent of the injury. In the same study, the high levels of IL-6 (more than $500 \mathrm{pg} / \mathrm{ml}$ ) have been correlated with multiple organ dysfunction and prolonged intubation [32]; these findings are consistent with other studies published by other investigators [33]. More research is to be done in order to correlate those findings with clinically important outcomes.

Since pulmonary function is disturbed, any patient at risk should be closely monitored for hypoxaemia using a pulse oxymetry and with regular ABGs samples [34]. Moreover early immobilisation of fractures by splinting reduces the incidence of FES; the risk is further reduced by surgical fixation and stabilisation of the fracture, especially compared to those treated conservatively. The necessity for early rigid fixation has been supported by many surgeons; Tachakra et al. [35] concluded to the following observations: 1) development of hypoxaemia in non-surgically treated patients with long-bone fractures; 2) occurrence of two distinctive episodes of FES in the same patient with multiple fractures; 3) appearance of skin petechiae pathognomonic of repeated episodes of embolisation. A large retrospective study published by Johnson et al. [36] revealed that the interval between the time of injury and fixation of the fracture is closely related to respiratory distress symptoms. In particular, among the 132 multi trauma patients that developed respiratory failure, the incidence of the syndrome was more than fivefold in those operated, for pelvic and long-bone fractures, 24 hours after the injury, than those who went to theatre after haemodynamic stabilisation. In the first case the incidence of FES is low, around 7\%, while in the delayed group the incidence is climbed up to $39 \%$.

Intramedullary nailing process and reaming techniques are known to produce increased intra-canal pressure followed by fat emboli [37] intravasation. A number of studies have assessed the effects of nail insertion with and without reaming the canal [38]-[40]. Reaming procedure is considered responsible for increasing medullary canal pressure; however studies have shown that unreamed nail insertion also results in an increase of the pressure [41]. Results conclude that there is no significant statistical difference in the rate of pulmonary responses between patients that were treated with reamed than those treated with unreamed nails. 
Another study compared the effects of reamed femoral nailing fixation versus plating fixation in poly-trauma patients with a concomitant head injury. Nailing had equivalent overall complications and functional results with plate fixation one year post-operatively [42].

Treating FES can be really challenging with uncertain results since there are no specific universally accepted protocols. Supportive care is of paramount importance and includes maintenance of adequate oxygenation and ventilation with positive and expiratory pressure (PEEP), haemodynamic stability, administration of blood products as clinically needed, hydration, thromboprophylaxis with low molecular heparins and adequate nutrition.

\section{Summary}

Fat embolism syndrome is a well-known severe condition that frequently presents mainly in trauma patients with one or multiple long bone fractures. Diagnosing the condition is in many cases demanding and can be missed by specialists with experience in the field of trauma. Diagnosis FES is mainly based on the clinical criteria proposed by Gurd in 1970, which has been slightly modified within those years. Despite advances in modern diagnostic imaging modalities and the abundant number of laboratory investigations, the diagnosis of FES remains clinical. Therefore, familiarisation with the condition, high clinical suspicion-especially in patients that meet the criteria for developing the syndrome - and finally meticulous and repetitive evaluation of the patient may lead to early diagnosis of the condition and rapid therapeutic response.

The authors have no conflict of interest.

No funding has been received for this work from the following organisations: National Institutes of Health (NIH); Wellcome Trust; the Howard Hughes Medical Institute (HHMI).

\section{References}

[1] Scuderi, C.S. (1943) The Present Status of Fat Embolism Syndrome. Bibliographic Review. International Surgical Digest, 18, 195- 215.

[2] Von Bergmann, E. (1873) Ein fall todlicher fettembolie. In: Wchenschr, B.K., Ed., Butterworth, London, 385.

[3] Gossling, H.R., Pellegrini Jr., V.D. (1982) Fat Embolism Syndrome: A Review of the Pathophysiology and Physiological Basis of Treatment. Clinical Orthopaedics and Related Research, 165, 68-82.

[4] Hofmann, S., Huemer, G. and Salzer, M. (1998) Pathophysiology and Management of the Fat Embolism Syndrome. Anaesthesia, 53, 35-37. http://dx.doi.org/10.1111/j.1365-2044.1998.tb15146.x

[5] Wenda, K., Runkel, M., Degruf, J. and Rutter, G. (1993) Pathogenesis and Clinical Relevance of Bone Marrow Embolism in Medullary Nailing-Demonstrated by Intraoperative Echocardiography. Injury, 24, S73-S81. http://dx.doi.org/10.1016/0020-1383(93)90011-T

[6] Pape, H.C., Dwenger, A., Grotz, M., et al. (1996) Does the Reamer Type Influence the Degree of Lung Disfunction after Femoral Nailing Following Severe Trauma? An Animal Study. Journal of Orthopaedic Trauma, 40, 980-984.

[7] Duwelius, P.J., Huckfeldt, R., Mullins, R.J., et al. (1997) The Effects of Femoral Intramedullary Reaming on Pulmonary Function in a Sheep Lung Model. American Journal of Bone and Joint Surgery, 79, 194-202.

[8] Barre, J., Lepouse, C. and Segal, P. (1997) Embolisation and Intramedullary Femoral Surgery. Revue de Chirurgie Orthopedique Reparatrice de l' Appareil Moteur, 83, 9-21.

[9] Arroyo, J.S., Garvin, K.L. and Mc Guire, M.H. (1994) Fatal Marrow Embolisation Following Porous-Coated Bipolar Hip Endoprosthesis. Journal of Arthroplasty, 9, 449-452. http://dx.doi.org/10.1016/0883-5403(94)90057-4

[10] King, E.G., Wagner Jr, W.W., Ashbaugh, D.G., Latham, L.P. and Halsey, D.R. (1971) Alterations in Pulmonary Microanatomy after Fat Embolism. Chest, 59, 524-530. http://dx.doi.org/10.1378/chest.59.5.524

[11] Pell, A.C., Hughes, D., Keating, J., Christie, J., Busuttil, A. and Sutherland, G.R. (1993) Brief Report: Fulminating Fat Embolism Syndrome Caused by Paradoxical Embolism through a Patient Foramen Ovale. New England Journal of Medicine, 329, 926-929. http://dx.doi.org/10.1056/NEJM199309233291305

[12] King, M.B. and Harmon, K.R. (1994) Unusual Forms of Pulmonary Embolism. Clinics in Chest Medicine, 15, 561580.

[13] Gurd, A.R. (1970) Fat Embolism: An Aid to Diagnosis. The Journal of Bone and Joint Surgery, 52, 732-737.

[14] Lindeque, B., Schoeman, H., Dommisse, G., Boeyens, M.C. and Vlok, A.L. (1987) Fat Embolism and the Fat Embolism Syndrome. The Journal of Bone and Joint Surgery, 69, 128-131.

[15] Schonfeld, S.A., Ploysongsan, Y., DiLisio, R., et al. (1983) Fat Embolism Prophylaxis with Corticosteroids. A Prospective Study in High Risk Patients. Annals of Internal Medicine, 99, 438-443. 
http://dx.doi.org/10.7326/0003-4819-99-4-438

[16] Burger, L.W., Dines, D.E. and Linscheid, R.L. (1974) Fat Embolism Syndrome in the Adult Respiratory Distress Syndrome. Mayo Clinic Proceedings, 49, 107-109.

[17] Bulger, E.M., Smith, D.G., Maier, R.V. and Jurkovich, G.J. (1997) Fat Embolism Syndrome. A 10 Year Review. Archives of Surgery, 132, 435-439. http://dx.doi.org/10.1001/archsurg.1997.01430280109019

[18] Gupta, A. and Reilly, C. (2007) Fat Embolism. Continuing Education in Anaesthesia Critical Care \& Pain, 7, $148-151$. http://dx.doi.org/10.1093/bjaceaccp/mkm027

[19] Butteriss, D.J., Mahad, D., Soh, C., Walls, T., Weir, D. and Birchall, D. (2006) Reversible Cytotoxic Cerebral Oedema in Cerebral Fat Embolism. American Journal of Neuroradiology, 27, 260-263.

[20] Meeke, R.I., Fitzpatrick, G.J. and Phela, D.M. (1987) Cerebral Oedema and Fat Embolism Syndrome. Intensive Care Medicine, 13, 291-292. http://dx.doi.org/10.1007/BF00265121

[21] Scopa, M., Magatti, M. and Rossitto, P. (1994) Neurologic Symptoms in Fat Embolism Syndrome: Case Report. Journal of Trauma, 36, 906-908. http://dx.doi.org/10.1097/00005373-199406000-00030

[22] Mellor, A. and Soni, N. (2001) Fat Embolism. Anaesthesia, 56, 145-154. http://dx.doi.org/10.1046/j.1365-2044.2001.01724.x

[23] Kaplan, R.P., Grant, J.N. and Kaufman, A.J. (1986) Dermatologic Features of the Fat Embolism Syndrome. Cutis, 38, 52-55.

[24] Tachakra, S.S. (1976) Distribution of Skin Petechiae in Fat Embolism Rash. The Lancet, 1, 284-285. http://dx.doi.org/10.1016/S0140-6736(76)91408-2

[25] Jaffe, F.A. (1994) Petechial Haemorrhages. A Review of Pathogenesis. American Journal of Forensic Medicine and Pathology, 15, 203-207. http://dx.doi.org/10.1097/00000433-199409000-00004

[26] Jones, J.P. (1993) Fat Embolism, Intravascular Coagulation and Osteonecrosis. Clinical Orthopaedics and Related Research, 292, 294-308.

[27] Van der Brande, F.G.J., Hellemans, S., De Schepper, A., et al. (2006) Post-Traumatic Severe Fat Embolism Syndrome with Uncommon CT Findings. Anaesthesia and Intensive Care, 34, 102-106.

[28] Styles, L.A., Schalkwijk, C.G., Aarsman, A.J., Vichinsky, E.P., Lubin, B.H. and Kuypers, F.A. (1996) Phospholipase A2 Levels in Acute Chest Syndrome of Sickle Cell Disease. Blood, 87, 2573-2578.

[29] White, T.O., Jenkins, P.J., Smith, R.D., et al. (2004) The Epidemiology of Post Traumatic Adult Respiratory Distress Syndrome. Journal of Bone and Joint Surgery, 86, 2366-2376.

[30] Peltier, L. (1969) Fat Embolism: A Current Concept. Clinical Orthopaedics and Related Research, 66, 241-253. http://dx.doi.org/10.1097/00003086-196909000-00028

[31] Pape, H.C., Schmidt, R.E., Rice, J., et al. (2000) Biochemical Changes after Trauma and Skeletal Surgery of the Lower Extremity: Quantification of the Operative Burden. Critical Care Medicine, 28, 3441-3448. http://dx.doi.org/10.1097/00003246-200010000-00012

[32] Pape, H.C., van Griensven, M., Rice, J., et al. (2001) Major Secondary Surgery in Blunt Trauma Patients and Perioperative Cytokine Liberation: Determination of the Clinical Relevance of Biochemical Markers. The Journal of Trauma, 50, 989-1000. http://dx.doi.org/10.1097/00005373-200106000-00004

[33] Patrick, D.A., Moore, F.A., Moore, E.E., et al. (1996) The Inflammatory Profile of Interleukin-6, Interleukin-8 and Soluble Intercellular Adhesion Molecule-1 in Post Injury Multiple Organ Failure. The American Journal of Surgery, 172, 425-429. http://dx.doi.org/10.1016/S0002-9610(96)00252-8

[34] Moed, B.R., Boyd, D.W. and Andring, R.E. (1993) Clinically Inapparent Hypoxemia after Skeletal Injury. The Use of Pulse Oxymetry as a Screening Method. Clinical Orthopaedics and Related Research, 293, 269-273.

[35] Tachakra, S.S., Potts, D. and Idowu, A. (1990) Early Operative Fracture Management of Patients with Multiple Injuries. British Journal of Surgery, 77, 1194. http://dx.doi.org/10.1002/bjs.1800771040

[36] Johnson, K.D., Cadambi, A. and Seibert, G.B. (1985) Incidence of Adult Respiratory Distress in Patients with Multiple Musculoskeletal Injuries: Effect of Early Operative Stabilisation of Fractures. The Journal of Trauma, 25, 375-384. http://dx.doi.org/10.1097/00005373-198505000-00001

[37] Christie, J., Robinson, C.M., Pell, A.C., et al. (1995) Transcardiac Echocardiography during Invasive Intramedullary Procedures. Journal of Bone and Joint Surgery, 77, 450-455.

[38] Anwar, I.A., Battistella, F.D., Neiman, R., et al. (2004) Femur Fractures and Lung Complications: A Prospective Randomised Study of Reaming. Clinical Orthopaedics and Related Research, 422, 71-76. http://dx.doi.org/10.1097/01.blo.0000129150.92270.19

[39] Heim, D., Schlegel, U. and Perren, S.M. (1993) Intramedullary Pressure in Reamed and Undreamed Nailing of the 
Femur and Tibia: An in Vitro Study in Intact, Human Bones. Injury, 24, S56-S63. http://dx.doi.org/10.1016/0020-1383(93)90008-T

[40] Schemitsch, E.H., Jain, R., Turchin, D.C., et al. (1997) Pulmonary Effects of Fixation of a Fracture with a Plate Compared with Intramedullary Nailing. A Canine Model of Fat Embolism and Fracture Fixation. Journal of Bone and Joint Surgery, 79, 984-996.

[41] Coles, R.E., Clements, F.M., Lardenoye, J.W., et al. (2000) Transesophageal Echocardiography in Quantification of Emboli during Femoral Nailing: Reamed versus Unreamed Techniques. Journal of the Southern Orthopaedic Association, 9, 98-104.

[42] Bhandari, M., Guyatt, G.H., Khera, V., et al. (2003) Operative Management of Lower Extremity Fractures in Patients with Head Injury. Clinical Orthopaedics and Related Research, 407, 187-198.

http://dx.doi.org/10.1097/00003086-200302000-00027 
Scientific Research Publishing (SCIRP) is one of the largest Open Access journal publishers. It is currently publishing more than 200 open access, online, peer-reviewed journals covering a wide range of academic disciplines. SCIRP serves the worldwide academic communities and contributes to the progress and application of science with its publication.

Other selected journals from SCIRP are listed as below. Submit your manuscript to us via either submit@scirp.org or Online Submission Portal.
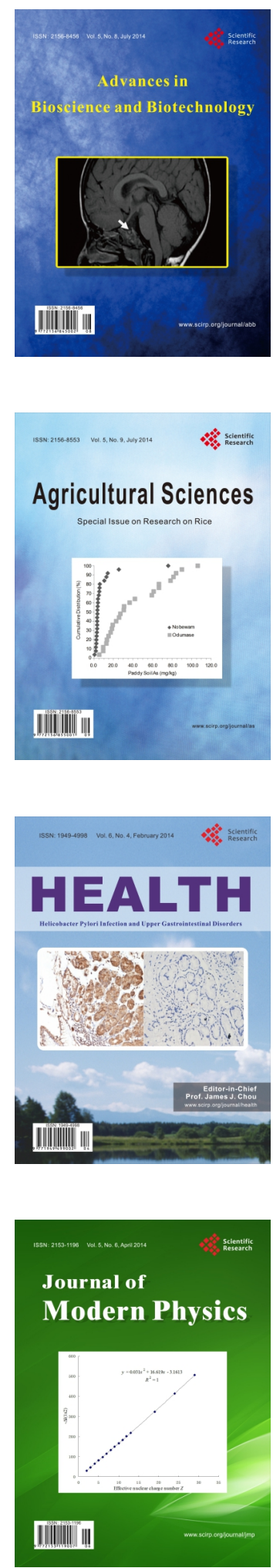
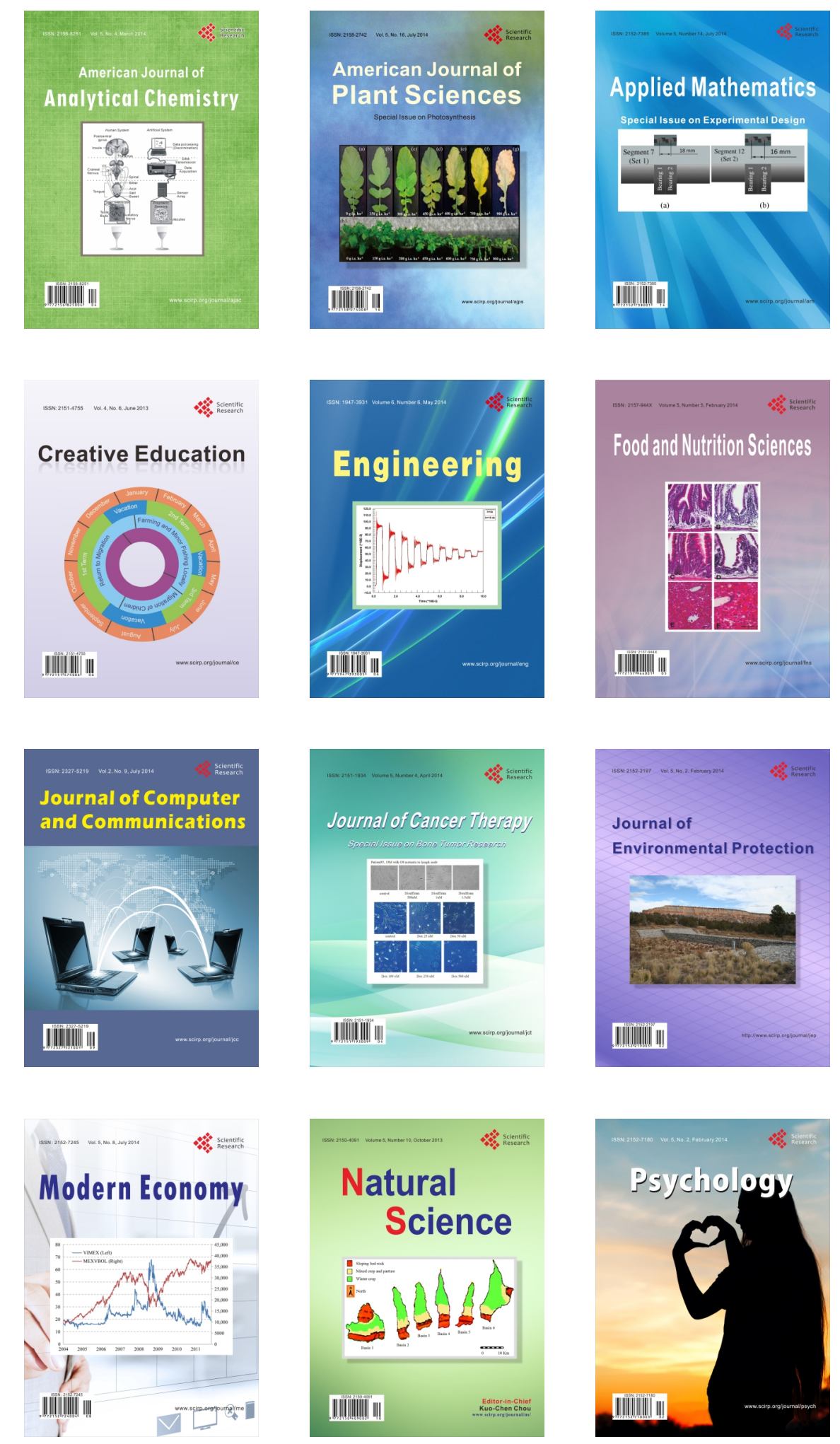